\title{
EL PAPEL DEL ACOMPAÑANTE EN LA CONSULTA MÉDICA DE ATENCIÓN PRIMARIA. ROLES Y EFECTOS SOCIALES
}

\author{
NIEVES HERNÁNDEZ FLORES \\ Universidad de Copenhague
}

\section{RESUMEN}

Este estudio trata del papel social y discursivo del acompañante del paciente en la consulta médica de atención primaria. Con un marco teórico y metodológico dentro de la sociopragmática cultural y el análisis del discurso, y mediante el análisis cualitativo de un corpus propio, el objetivo es mostrar que el acompañante (un familiar) representa roles que son confirmados de acuerdo a expectativas sociales, pero también negociados y construidos por medios discursivos en la interacción concreta. Estos roles del acompañante dependen del comportamiento de los otros dos participantes (el médico y el paciente) y se relacionan con el deseo de confirmar y proteger la imagen social. Los resultados confirman las conclusiones de estudios previos en cuanto a la multiplicidad de roles desempeñados por el acompañante y sus características, vinculadas a asuntos prácticos e instrumentales como: enfermero, cuidador o secretario. Pero además se revelan otros roles vinculados a aspectos sociales y psicológicos: el rol de dinamizador social, que mediante manifestaciones discursivas no necesariamente relacionadas con asuntos médicos facilita la comunicación y reafirma la imagen afiliativa de los interactuantes, y el rol de asistente emocional, de carácter psicológico, que mediante el sostén emocional refuerza la afiliación.

PALABRAS CLAVE: consulta de atención primaria, interacción tripartita, acompañante, roles, imagen social.

\section{ABSTRACT}

This study deals with the social and communicative role of the patient's attendant -a relative- in primary care medical consultation. The theoretical and methodological background is based on cultural sociopragmatics and discourse analysis. The qualitative data analysis aims to show that the attendant's roles are confirmed in keeping with social expectations but also negotiated and constructed in the communicative interaction. The attendant's roles depend on 
the other participants' -doctor and patient- behaviors and these roles are related to the speakers' face wants. The results confirm previous studies about the attendant's multiplicity of roles and about the instrumental and practical characteristics of these roles, such as nurse, care-giver and secretary. Furthermore, other socially and psychologically related roles have been identified in the analysis. The role of social 'driver' contributes to the positive development of the social and communicative interaction -for example by introducing topics that are not related to medical topics. The role of emotional assistant means an emotional support to the patient. Both types of roles reinforce the interactants' wants of affiliation face.

KEYWORDS: primary care medical consultation, tripartite interaction, patient's attendant, roles, face.

\section{INTRODUCCIÓN}

Como sabemos, la comunicación en situaciones de consulta médica es básicamente de carácter bipartito: un/una paciente interacciona con un/ una médico en un espacio delimitado con la finalidad de obtener atención sanitaria. Pero en ocasiones el médico está asistido por otra persona (un médico en prácticas, un enfermero), o bien el paciente acude con un acompañante, normalmente un familiar o un asistente social, dando lugar a una comunicación de carácter tripartito.

La investigación pragmática y discursiva de la interacción con fines médicos se ha ocupado principalmente de las situaciones con dos interactuantes, mientras que la presencia de un tercero no ha sido tan estudiada (cf. Madfes, 2006; Cordella, 2011a, 2011b), y cuando así ha sido el centro de estudio lo ha ocupado preferentemente la consulta pediátrica (en que el paciente, un niño, es acompañado por un progenitor) y la geriátrica (en que el paciente de edad avanzada acude con una persona cercana) (Cordella, 2011a, 2011b; Pennbrant, 2013). La investigación reciente en inglés (cf. Mullany, 2009: 3) ha propuesto superar el habitual enfoque en la interacción médico-paciente en situación de consulta para incluir nuevos géneros comunicativos como la consulta por e-mail o los foros de internet, así como desplazar el habitual enfoque en la figura del médico para incluir situaciones con una variedad de interlocutores que de alguna forma participan en el proceso de la intervención médica, entre los cuales estaría el acompañante del paciente.

Este trabajo tiene como objetivo estudiar el papel del acompañante familiar del paciente en consultas de atención primaria. En esas situaciones, tanto en estudios médicos de comunicación como en estudios pragmáticos y discursivos, se ha destacado la multiplicidad de funciones que este desempeña, lo que viene determinado por factores sociales (la relación que 
lo vincula con el paciente); situacionales (su participación en la asistencia y cuidado de este, como vigilar la correcta toma de medicamentos); y discursivos (su intervención comunicativa durante la consulta, como aportar información al facultativo sobre el estado del paciente). Concretamente, en estudios médicos sobre comunicación, el enfoque se ha situado en las ventajas e inconvenientes de la presencia del acompañante en la consulta en cuanto a la duración de la misma y a la relevancia de su colaboración en la búsqueda de un correcto diagnóstico y prescripción (cf. López GarcíaRamos et al., 2009; Turabián y Pérez Franco, 2015; Turabián et al., 2016). Por su parte, en estudios pragmáticos y discursivos, el enfoque se ha localizado en la configuración discursiva de estos encuentros (la intervención del acompañante en la interacción) y los roles adoptados de acuerdo con el comportamiento comunicativo desempeñado durante la consulta, identificándose una serie de roles cuya aparición es frecuente que se repita en diferentes corpus, tales como la función de asistencia sanitaria y ayuda con lo práctico, o la intermediación con el facultativo (cf. Madfes, 2006; Cordella, 2011 a y 2011 b).

En este trabajo voy a partir de estudios anteriores que han tratado el papel social y discursivo del acompañante en la consulta médica para adentrarme en el análisis de un corpus propio (De Oliveira y Hernández Flores, 2014) con el objetivo de profundizar en los diferentes roles adoptados por este hablante a lo largo de la interacción. Parto de la premisa de que los roles están en parte configurados socialmente de acuerdo con lo esperado en la comunidad sociocultural específica para este tipo de encuentro comunicativo y que, por tanto, es esperable que se repitan en interacciones particulares. La hipótesis es que estos roles, sin embargo, son confirmados, negociados y coconstruidos por medios discursivos en la interacción concreta, dando lugar a roles específicos para ese encuentro comunicativo. Estos roles se constituirían no solo por los comportamientos comunicativos desempeñados por el acompañante, sino que dependen del comportamiento de los otros dos participantes (el médico y el paciente). Además, esta configuración de roles estaría relacionada con los deseos de confirmación y protección de la imagen social ('face', Goffman, 1967) de los hablantes, es decir, con el deseo de confirmar y proteger su autoestima en encuentros comunicativos, un tema ampliamente tratado por estudios de interacción médica bipartita (Madfes, 2003; Cepeda, 2005, 2006, 2009; Hernández López, 2009, 2010a, 2010b), pero no tanto en las de carácter tripartito.

El estudio consistirá en un análisis cualitativo de extractos proveniente de un corpus de consultas médicas grabado en audio en un municipio de la región de Galicia, España (De Oliveira y Hernández Flores, 2014). La base teórica y metodológica proviene de la sociopragmática cultural y el análisis del discurso. 


\section{LAS CONSULTAS TRIPARTITAS EN LA COMUNICACIÓN MÉDICO-PACIENTE}

Desde una perspectiva médica, el interés en el papel del acompañante en la consulta tiene que ver con el objetivo de lograr una comunicación efectiva con el paciente. Además, se reconoce su influencia en la situación anímica de este e, incluso, en sus posibilidades de recuperación (cf. Turabián et al., 2016). Por estos motivos los resultados de estudios médicos coinciden en las ventajas de la presencia del acompañante, a pesar de que esto suponga una ligera mayor inversión de tiempo (cf. López García Ramos et al., 2009). Esta ventaja es especialmente relevante en la consulta de atención primaria debido a la importancia otorgada por los médicos de cabecera a la respuesta del paciente a la enfermedad, por encima de la enfermedad en sí, es decir, a la intervención de factores psicológicos y sociales en la curación. En ese sentido, el conocimiento de la personalidad y del medio socio-afectivo del paciente, así como la posible relación de estos factores con la presentación de síntomas, es tan importante como la dolencia en sí, y en esa toma de conocimiento el acompañante juega un papel relevante (Turabián et al., 2016: 1). Este papel se traduce en dos perfiles principales de acompañante, categorizados desde la perspectiva y expectativas del médico: el colaborador y el no colaborador (Turabián et al, 2016: 2). El primero se caracteriza por la ayuda respetuosa al doctor, la aportación de información relevante, su toma de responsabilidad en cuanto al proceso terapéutico, y su función de mediador con el paciente. El segundo es contemplado en varios grados según su mayor o menor involucramiento en la consulta, que puede ir desde la intervención excesiva que anula la del paciente a la presentación de estados psicológicos relacionados con la culpabilidad o con sus propios sentimientos, entre otros. Esta caracterización supone que aun reconociendo las ventajas de la presencia del acompañante en la consulta, se destaquen los retos comunicativos que pueden suponer para el facultativo; por ello se aboga por realizar estudios que aporten directrices sobre cómo manejar una consulta tripartita (Turabián y López Franco, 2015; Turabián et al., 2016).

Desde la perspectiva pragmático-discursiva, en consultas de carácter tripartito el interés en la figura del acompañante ha residido en observar funciones como la de informar al médico, relevar al paciente en momentos donde este puede estar poco activo por razones de salud y, en general, apoyar su bienestar. En corpus del español estos aspectos han sido observados mediante el análisis de la configuración de la estructura interaccional, en concreto la función de receptor o destinatario (directo o indirecto) que puede otorgarle el médico (Madfes, 2006), la asimetría interaccional producida (Madfes, 2006; Cordella, 2011a), y las estrategias lingüísticas empleadas (Cordella, 2011a). Por otra parte, también se ha realizado iden- 
tificación y descripción de los roles desempeñados por el acompañante (Madfes, 2009; Cordella, 2011a y 2011b).

Con respecto a la construcción interaccional y a las estrategias lingüísticas empleadas, señala Madfes (2006) que en la interacción trilógica la recepción es más borrosa, fluctuante, imprevisible, conflictiva y solapada de lo que es en interacciones dialógicas. Así, por una parte, el médico puede decidir (a priori o a lo largo de la interacción) si el destinatario es solo el paciente (y el acompañante el destinatario secundario), o si paciente y acompañante conforman una unidad receptora (papel de codestinatario directo, junto con el paciente) (Madfes, 2006: 170). La función comunicativa del acompañante puede ser pasiva o activa, y es ese comportamiento el que en muchos casos define funciones y categorías. La pasiva corresponde a una persona que se mantiene en silencio durante la consulta, el acompañante silencioso, mientras que una función activa consiste en completar, desarrollar o corregir las respuestas del paciente (Madfes, 2006: 172). Este es el caso del acompañante portavoz, que sustituye al paciente en algunas funciones comunicativas como informar al médico, un tipo de intrusión que Madfes considera cooperativa (2006: 175), a diferencia de las intervenciones del acompañante dominante, quien intenta dirigir al médico. Pero como categorías de roles principales, hay dos que se señalan en el trabajo de Madfes: el informante, que completa la tarea comunicativa del paciente (Madfes, 2006) y la cuidadora (la autora lo identifica con el sexo femenino), un rol validado por el médico cuya principal actuación es administrar las medicinas y organizar el tipo de vida del paciente. Como vemos, se trata de dos tipos de roles caracterizados por su objetivo funcional (ayudar con la comunicación) y con la atención diaria, comportamientos que suponen un soporte tanto para el paciente como para el médico.

Por su parte, Cordella (2011a y 2011b) desglosa estos dos papeles principales en una clasificación más extensa que incluye siete tipos de roles que pueden aparecer en los diferentes turnos de habla, y que se definen según las funciones sociales desempeñadas en relación con el paciente, y según el comportamiento comunicativo con este y con el médico. Los roles identificados por Cordella (2011b: 341) son: el asistente familiar de salud quien, entre otras funciones, confirma y ratifica información al doctor y, además, solicita pruebas médicas; el compañero, que complementa y apoya al propio paciente; el consejero de salud, que informa al médico de lo que observa y de cuestiones de interés, mostrando cierta competencia sanitaria; el comunicador de aspectos sociales, que revela temas familiares y personales del paciente; el reportero, un portavoz del paciente que toma la iniciativa sin que le pregunten; la (sic) secretaria, que se ocupa de las cuestiones administrativas; y el asistente de finanzas, quien trata las cuestiones económicas de la salud. 
En lo referente al papel de los hablantes durante la interacción, Madfes (2006: 169) recoge la noción de marco participativo de Kerbrat Orecchioni, para tener en cuenta tanto el nivel de secuencias concretas como el nivel global de la interacción, es decir, el cotexto. Por otra parte, al estudio de la construcción interaccional entre los tres hablantes, Madfes añade la historia previa de interacciones, es decir, el conocimiento anterior a la consulta donde se produce la comunicación entre el médico de cabecera y el paciente (Madfes, 2006: 183). Este estudio extrainteraccional es también considerado por Cordella (2011a) en los casos de enfermedades de larga duración, pues los roles, dependiendo de la naturaleza y evolución de la enfermedad, se desarrollan también fuera de la interacción de la consulta con el médico. Sin embargo, en mi opinión, estos aspectos, que sin duda son muy relevantes para la comprensión de las relaciones construidas y de los roles adoptados, quedan muchas veces fuera del alcance del investigador, quien solo dispone de lo que efectivamente se dice en las interacciones de la consulta.

\section{LA IMAGEN SOCIAL EN LA CONSULTA MÉDICA}

Como es sabido, toda comunicación humana, aparte de objetivos como la transmisión de información o el tratar de conseguir algo (los fines transaccionales) tiene, en mayor o menor medida, un componente social. El componente social es evidente en la interacción de carácter informal o coloquial, pero también en interacciones profesionales o de carácter institucional, como es el caso que nos ocupa. Por ello, cuestiones sociales que contribuyen a mantener una comunicación lograda para los fines de servicio médico -como la negociación del poder interaccional, la adopción de roles en situaciones específicas, los deseos de imagen social durante la interacción y la realización de comportamientos de cortesía en el contexto específico donde se desarrolla la interacción- han constituido temas de interés en la investigación pragmática de la interacción médico-paciente.

La imagen social ('face') es un concepto sociopragmático que ha sido ampliamente estudiado empíricamente en todo tipo de discurso. Cuestiones como el efecto social que producen los comportamientos comunicativos (cf. Bravo, 2005), especialmente el efecto positivo para la imagen social de los interactuantes que supone la cortesía, han sido tratadas en la interacción médico-paciente (por ejemplo, Cepeda, 2005, 2006, 2009; Hernández López, 2009, 2010a, 2010b), a veces junto con comportamientos de autoimagen (cf. Hernández Flores, 2013, 2016; Hernández Flores y Rodríguez Tembrás, 2016; Rodríguez Tembrás, en prensa), pero también en 
cuanto a las amenazas a la imagen que se pueden producir en la interacción y a las relaciones de poder y de desigualdad (Madfes, 2003; Hernández López, 2009, 2010a).

La cuestión de la imagen también se ha estudiado en actos de habla concretos como el consejo médico (Harrison y Barlow, 2009; Hernández Flores y Rodríguez Tembrás, en prensa) y los cumplidos (Backhaus, 2009), así como en el tratamiento de temas embarazosos mediante la minimización de la imposición (Brown y Crawford, 2009). También en relación con la imagen social se han analizado las estrategias lingüísticas que señalan comportamiento de empatía (Cepeda, 2006); los recursos de atenuación e intensificación (Cepeda, 2009), del humor (Hernández López, 2010b) y de la alternancia de lenguas (Rodríguez Tembrás, 2016). Por otra parte, al tratar de la imagen social se han descrito las características, de naturaleza cultural, que se presentan en la situación médica (Madfes, 2003; Hernández López, 2010a; Hernández Flores, 2016; Rodríguez Tembrás, 2016), relacionándose estas características con las de los roles desempeñados por los interactuantes (la imagen del rol, Bravo, 2002: 145).

\section{Metodología y datos}

Los datos utilizados para el presente estudio provienen de un corpus en audio de consultas médicas en un centro de atención primaria en un municipio gallego (De Oliveira y Hernández Flores, 2014) ${ }^{1}$. Se ha realizado una primera selección de datos usando el criterio de consultas con acompañante familiar, de donde se han elegido aquellas donde los participantes hablan principalmente en castellano ${ }^{2}$. Tras una primera revisión de estas consultas, se han identificado y descrito cuatro tipos básicos del rol de acompañante (ver 4.1.), siendo dos de ellos los elegidos como objeto de estudio. A partir de ahí, se han seleccionado dos consultas de dos médicos diferentes, con interés para el análisis según los criterios explicados. El análisis, de tipo cualitativo, se ocupa de la consulta completa y de algunas secuencias seleccionadas.

${ }^{1}$ La recogida de datos fue aprobada por el Consejo Ético de Galicia y las grabaciones consentidas por los hablantes para su uso científico. En las transcripciones se han cambiado los nombres y apellidos, así como cualquier otro dato identificativo de los participantes. Agradezco al Consejo Ético de Galicia, al personal del centro médico y a los pacientes su colaboración y facilitación de la recogida de datos.

${ }^{2}$ El corpus consta de consultas en gallego, en castellano y en ambas lenguas, siendo habitual que en ocasiones los hablantes usen cada uno una lengua, sin que esto interfiera en la comunicación, como es propio del bilingüismo en la región de Galicia. 


\section{ANÁlisis}

\subsection{Selección de datos e identificación de roles generales}

En una visión general del corpus, en lo referente a la participación discursiva del acompañante en relación con el paciente en la consulta médica, los datos muestran cuatro tipos generales que responden a una mayor o menor gradación de participación:

a) Alta participación de $\mathrm{P}$ y baja de $\mathrm{A}^{3}$. Se trata de pacientes autónomos donde A apenas interviene. El perfil de este corresponde con las denominaciones en la bibliografía de acompañante silencioso (Madfes, 2006: 174), acompañante pasivo (Cordella, 2011a: 473; Turabián y Franco, 2015: 210), y acompañante observador (Turabián y Franco, 2015: 210).

b) Baja participación de $\mathrm{P}$ y alta de A. Suele tratarse de pacientes con alguna limitación (enfermedad o edad, tanto elevada como menor), que en consulta son prácticamente reemplazados por A. Este perfil corresponde con el acompañante portavoz (Madfes, 2006: 175; Cordella, 2011a: 473).

c) Alta participación de $\mathrm{P}$, junto con actividad comunicativa de A.

d) Participación activa de $\mathrm{P}$, pero es A quien domina comunicativamente la consulta.

Para los fines de este trabajo, me fijaré en estos dos últimos casos, los de pacientes y acompañantes activos, pues se trata de roles sociales y discursivos interdependientes. En concreto, en los casos observados en el corpus (18 consultas en castellano de pacientes acompañados, repartidos entre cinco médicos) estas son las características de los respectivos roles:

- $\quad$ P activo y A activo. El acompañante añade, matiza o corrige la información que da el paciente, como el tipo de medicamento tomado o aspectos del historial médico. Corresponde con el rol de acompañante informante (Madfes, 2006: 183) o de tipo colaborador (Turabián y Franco, 2015: 210). En estas situaciones, y a pesar del papel relevante de A, $\mathrm{P}$ no deja de intervenir y de mantener las riendas de la comunicación con $\mathrm{M}$. En el presente trabajo se denominará este rol acompañante coparticipativo por su papel activo en la interacción junto con los otros hablantes.

- $\quad$ P activo (o moderadamente activo) y A muy activo. Aunque P participa, es A quien lleva el peso de la interacción (toma iniciativas,

${ }^{3}$ En lo que sigue, se usarán las abreviaturas: P, por el paciente; A, por el acompañante; y M, por el médico. 
maneja los temas, pregunta, responde), y desempeña una mayor variedad de funciones, tanto de cara al médico (informar, pedir información, solicitar pruebas, recetas o citas, entre otras) como al paciente (explicarle la información del médico, aconsejarle o recordarle cuestiones prácticas), por lo que en este trabajo será denominado acompañante protagonista. En ocasiones, además de complementar a $\mathrm{P}$, lo sustituye, funcionando entonces como acompañante portavoz (Madfes, 2006), o interviniendo en las negociaciones con $\mathrm{M}$, a veces por iniciativa propia (el acompañante reportero, Cordella, 2011a) pudiendo entonces ocupar el lugar de P, un tipo específico de rol de alto protagonismo que se ha considerado intrusivo (Turabián y Franco, 2015: 210).

En los subapartados siguientes se analizarán dos consultas que presentan, respectivamente, estos dos tipos de acompañantes.

\subsection{Paciente activo con acompañante activo. El acompañante coparticipativo}

Este tipo de interacción se mostrará mediante el análisis de una consulta de 16 minutos y 43 segundos de duración, entre un médico de cabecera (el doctor Castro), su paciente (una mujer de 71 años llamada Gloria) y el acompañante (su marido). Gloria acude para recibir seguimiento médico sobre un cólico biliar que la tuvo ingresada en un hospital en fechas recientes. Además, es reconocida por el doctor porque tose repetidamente durante la consulta. Esta está estructurada de la siguiente manera: preámbulos; pregunta sobre el motivo de la visita; interrogatorio médico; prescripción de pruebas; narración de $\mathrm{P}$ y A sobre asuntos de su familia y un viaje reciente (con participación de $\mathrm{M}$ ); reconocimiento físico de $\mathrm{P}$ paralelo a una nueva narración de A sobre su viaje y sobre un partido de fútbol (también con participación de M); preguntas e información sobre nuevas pruebas y medicación; pregunta de A a M sobre sus vacaciones, lo que da pie a una discusión sobre el tema del derecho al tiempo de asueto; y finalmente, agradecimiento y despedida.

Desde el punto de vista de la participación en la interacción, de un total de los 196 turnos de que consta la transcripción básica ${ }^{4}, 78$ son ocupados por M, 61 por A y 57 por $\mathrm{P}$, de forma que acompañante y paciente

\footnotetext{
${ }^{4}$ Con ello me refiero a una primera transcripción de la consulta no detallada, es decir, de contenidos de lo dicho con los turnos identificables en las primeras audiciones. Por otra parte, las secuencias extraídas para análisis han sido transcritas de forma completa siguiendo los procedimientos habituales de la investigación pragmática, en concreto utilizando el sistema de transcripción del grupo Val.Es.Co. (<http://www.uv.es/valesco/sistema.pdf $>)$.
} 
tienen un número de turnos parecidos. Las intervenciones de A son fundamentalmente para preguntar e informar al médico, pero también para corroborar la información de la paciente. En concreto, son características del papel discursivo de A en la interacción total: la toma de iniciativa en el tratamiento de temas o explicaciones relacionados con la consulta (sobre todo de carácter práctico); la continuación de la información que da $\mathrm{P}$ al médico y la adhesión al discurso iniciado por ella (completándolo o matizándolo); el desarrollo junto a $\mathrm{P}$ de dos temas personales no relacionados con la consulta médica, que fueron iniciados por ella, y en los que también participa M (los temas son el cuidado de los nietos del matrimonio y el partido de fútbol que vieron televisado en Barcelona), así como un tema de discusión (la cantidad de días de vacaciones de que dispone $\mathrm{M}$ ) iniciada por A. Desde un punto de vista interaccional, se observa que A finaliza las frases de $\mathrm{M}$ en dos ocasiones, repite (estructura ecoica) las palabras de $\mathrm{P}$ en una ocasión e introduce en otra una opinión médica en un turno que por rol habría correspondido a M.

Se trata, en suma, de un comportamiento discursivo activo que no acapara el papel protagonista de $\mathrm{P}$, pues de hecho es ella quien responde a las

\section{TABLA 1. Roles identificados en el comportamiento comunicativo} del acompañante coparticipativo

\begin{tabular}{|l|l|l|}
\hline \multicolumn{1}{|c|}{ Tipos de roles } & \multicolumn{1}{c|}{$\begin{array}{c}\text { Ejemplos } \\
\text { de comportamiento }\end{array}$} & $\begin{array}{c}\text { Número de ocasiones } \\
\text { en que se realiza } \\
\text { el comportamiento }\end{array}$ \\
\hline Secretario & $\begin{array}{l}\text { - establecimiento de citas } \\
- \text { documentación necesaria }\end{array}$ & 7 \\
\hline $\begin{array}{l}\text { Enfermero } \\
\text { y cuidador }\end{array}$ & $\begin{array}{l}\text { - informar de pruebas } \\
\text { anteriores } \\
-\begin{array}{l}\text { preguntar por resultados } \\
\text { de análisis o por toma de } \\
\text { medicación }\end{array}\end{array}$ & 9 \\
\hline $\begin{array}{l}\text { Conocedor } \\
\text { de temas médicos }\end{array}$ & $\begin{array}{l}\text { - manifestación } \\
\text { de su conocimiento de } \\
\text { la enfermedad } \\
\text { preguntas en torno } \\
\text { a la enfermedad }\end{array}$ & $\begin{array}{l}3 \text { (2 como } \\
\text { complemento } \\
\text { del explicación } \\
\text { del médico) }\end{array}$ \\
\hline Dinamizador social & $\begin{array}{l}\text { narración de hechos } \\
\text { junto con M y P } \\
- \text { charla con M sobre sus } \\
\text { condiciones vacacionales }\end{array}$ & 3 \\
\hline
\end{tabular}


preguntas de M y quien explica los síntomas, y que tampoco interfiere en el papel del médico. A pesar de su toma de iniciativa y de que en algunos momentos reemplace a los otros dos hablantes en turnos que se esperaría de estos, son más abundantes los casos en que A coconstruye su discurso tanto con $\mathrm{P}$ como con M. Esta coconstrucción discursiva muestra una serie de roles de A que se recogen en la tabla 1.

Varios de estos roles aparecen en la siguiente secuencia, que corresponde al comienzo de la consulta.

(1) $[\text { Castro 20A }]^{5}$

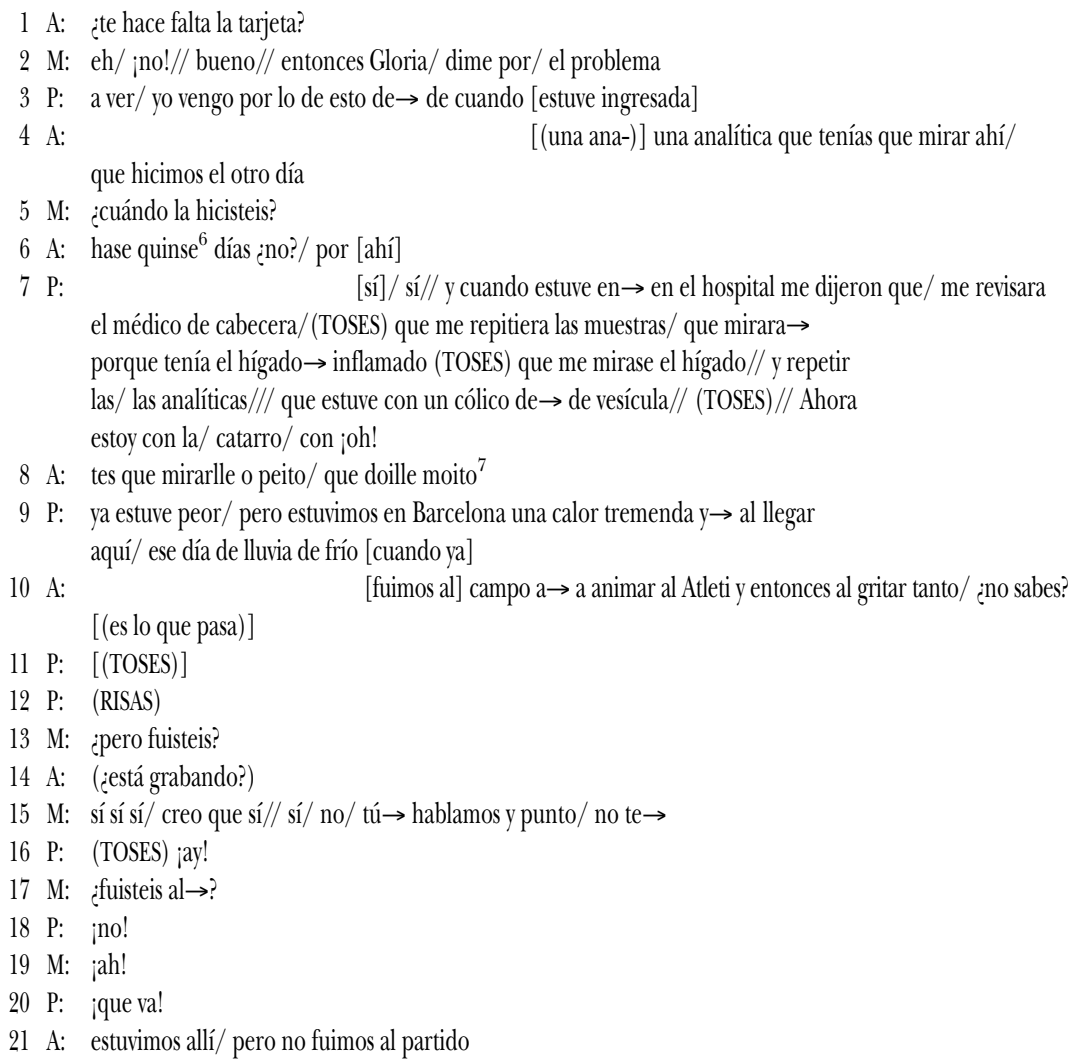

Este extracto sirve de muestra de los diferentes roles manifestados por A en la interacción comunicativa y su interrelación con las intervenciones

\footnotetext{
${ }^{5}$ Durante toda la secuencia se escucha el teclear del ordenador del doctor Castro.

${ }^{6}$ Pronunciado con seseo, propio de algunas zonas de Galicia.

7 En gallego, 'tienes que mirarle el pecho, que le duele mucho'.
} 
de sus interlocutores. A adopta en esta secuencia tres roles: un rol funcional de secretario, cuando ofrece la tarjeta sanitaria a M (turno 1) y se interesa por saber si la grabación para la presente investigación, cuya autorización acaba de firmar su mujer, está en marcha (turno 14); el rol de enfermero/cuidador, al completar la información que ha dado P sobre el motivo de la consulta (turno 4), y al solicitar, como consecuencia de la tos de $\mathrm{P}$, una exploración de bronquios (turno 8); pero, además, un rol no mencionado en la literatura del tema, el que denomino dinamizador social (turnos 10 y 16) donde bromea sobre el motivo de la tos de su mujer (sus gritos durante un partido de fútbol al que en realidad no asistieron), una intervención innecesaria para los fines de la interacción médica que, sin embargo, funciona como estrategia afiliativa entre los interactuantes mediante una desviación del tema médico hacia un tema personal (un viaje) realizado con humor (la conocida retranca -ironía- gallega). El cambio de código lingüístico al gallego por parte de A en su petición a M de que haga un reconocimiento a $\mathrm{P}$ (turno 8 ), supone un nuevo recurso afiliativo para una petición realizada mediante una estrategia directa (la perífrasis de obligación tener que), en ausencia de otros recursos corteses esperables en solicitudes a los médicos ${ }^{8}$. La iniciativa afiliativa de A es refrendada por las respuestas de M (turnos 13, 17 y 19) y de P (turno 18), y continuará a lo largo de la consulta en tres ocasiones más en que se repite esta estructura de conarración entre A y $\mathrm{P}$ de asuntos privados no relacionados con el tema médico que los ocupa, y en los que el mismo médico se involucra. Este mismo refrendo se observa en el hecho de que $M$ pasa de hacer destinataria de su intervención comunicativa a $\mathrm{P}$ (ver el uso de la segunda persona del singular y del vocativo en el turno 2: Gloria / dime) a hacer codestinatario a A en su turno siguiente (5, con el uso de la segunda persona del plural, hicisteis) en una pregunta sobre una prueba médica que solo se hizo $\mathrm{P}$, confirmando así al acompañante en su rol de enfermero/cuidador.

La siguiente secuencia sirve para confirmar la coconstrucción discursiva de esta consulta, así como para identificar un nuevo rol de A: el de conocedor de temas médicos.

(2) [Castro 20A]

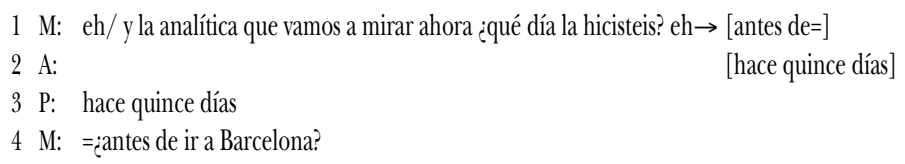

${ }^{8}$ Sobre la relación del cambio de código lingüístico con actividades de cortesía, ver el estudio de Rodríguez Tembrás (2016) sobre la alternancia castellano-gallego en este mismo corpus. 


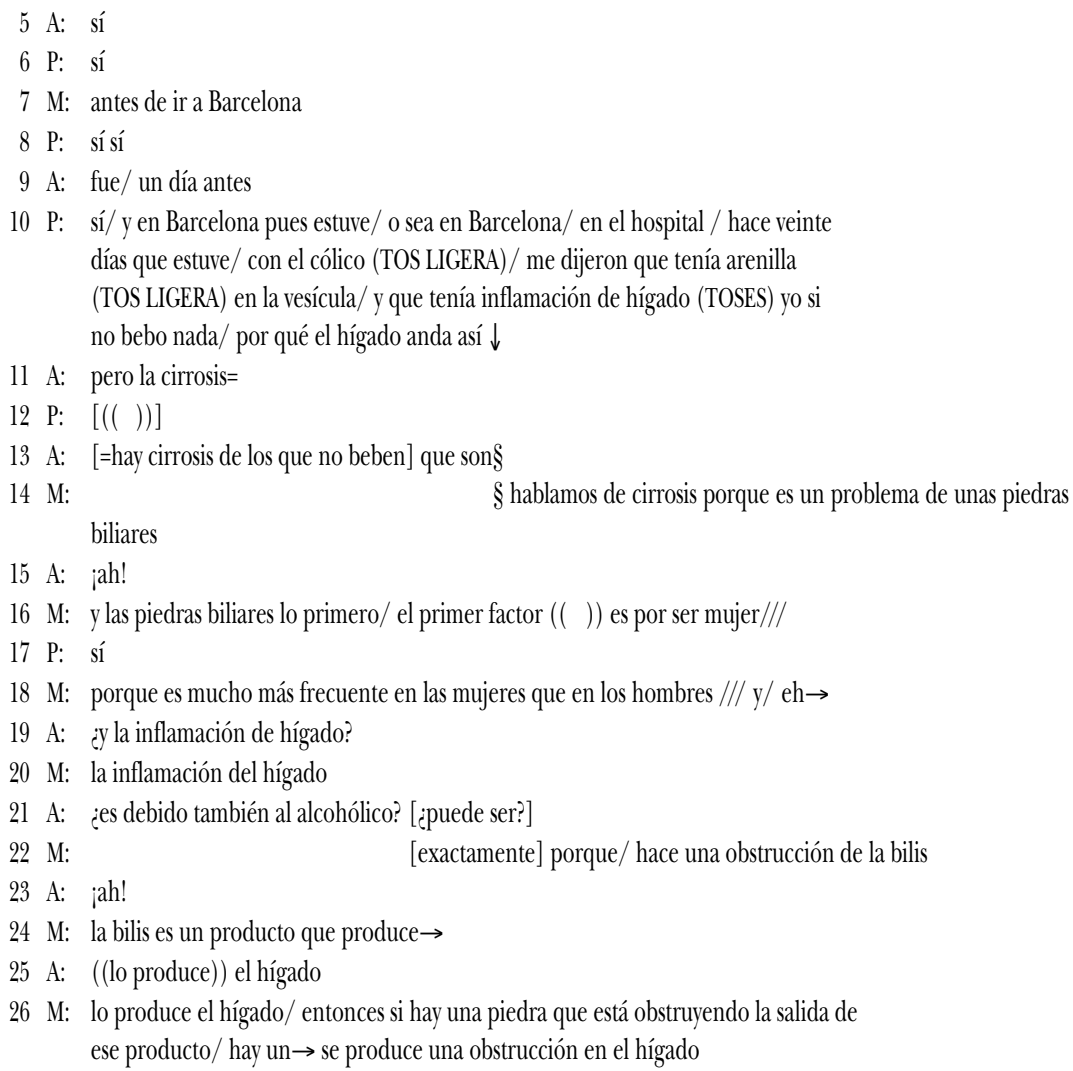

Esta secuencia muestra la colaboración del acompañante con la paciente en la facilitación de información de tipo práctico (rol de secretario, en los turnos 2, 5 y 9), en concreto de las fechas en que se hizo las pruebas médicas. Además, A activa un rol de conocedor de temas médicos dando una información que en principio correspondería dar al doctor, introduciendo un tema médico, la cirrosis (turno 11), que es retomado con una explicación especializada por M en el turno siguiente (14), es desarrollado tras una pregunta de A sobre el hígado ( 19 y 21), es completado por A (en 25) y corroborado por M (26). Se trata, en suma, de una clara muestra de coconstrucción discursiva entre los tres interactuantes donde la participación del acompañante facilita la información médica necesaria para que la paciente entienda la naturaleza de su enfermedad, es decir, un comportamiento participativo que beneficia el discurrir interaccional y contribuye a una consecución positiva de la consulta.

En definitiva, estamos ante un tipo de interacción con acompañante donde este es muy activo y desempeña diferentes roles vinculados a la si- 
tuación. A manifiesta sus opiniones y solicita servicios a M de forma directa, siendo a lo largo de su intervención escasos los recursos comunicativos asociados con la cortesía. Sin embargo, el uso de otras estrategias observables en el nivel macrodiscursivo (el tratamiento de temas que fomentan la socialización, el humor o el cambio de código al gallego), y la reacción de sus interlocutores ( $\mathrm{M}$ y P), quienes parecen aceptar e incluso participan en el tipo de interacción que A propone, indica que estamos ante un caso de un rol de acompañante que resulta constructivo y beneficioso para el buen discurrir y el logro final de la consulta médica.

\subsection{Paciente activo (o medianamente activo) con acompañante muy activo. El acompañante protagonista}

En la siguiente consulta médica se repiten algunos de los roles identificados en la interacción anterior, si bien aquí el paciente se retrae y la acompañante acapara un mayor papel en todo momento, de ahí la denominación de esta de acompañante protagonista. La consulta tiene una duración de 16 minutos y 4 segundos, y hay tres participantes: la doctora Núñez, el paciente (Juan Antonio, de 68 años) y la acompañante (su esposa). El motivo de la visita es recibir los resultados de unos análisis de próstata (PSA) que resultan positivos. Además, Juan Antonio padece de párkinson. La estructura de la consulta es la siguiente: preámbulos; explicación de los resultados de las pruebas; prescripción de nuevas pruebas; interrogatorio médico; interrupción de la consulta por parte de la médica (sale un momento), que es aprovechada por A y $\mathrm{P}$ para tratar unos asuntos prácticos; prescripción de una prueba inmediata en el mismo centro médico; preguntas de A sobre medicación anteriormente recetada; y despedida provisional (tienen que volver después de realizar la prueba).

En este encuentro comunicativo llama la atención que es A quien reacciona tanto a la información de los preocupantes resultados de los análisis como a la prescripción de nuevas pruebas, y es A quien principalmente lleva la comunicación con $\mathrm{M}$ en las diferentes fases de la consulta. De hecho, en cuanto a número de turnos, de un total de 186 contabilizados en la transcripción básica, $\mathrm{P}$ solo ocupa 28 frente a los 81 turnos de A, también por encima de los turnos de M (77).

El comportamiento comunicativo de A consiste principalmente en interaccionar con $\mathrm{M}$ : responde a sus preguntas en lugar de $\mathrm{P}$ (también en cuanto a los síntomas experimentados por este); toma la iniciativa a la hora de preguntar, comenta con M los análisis y la situación, y refrenda lo que dice $M$ (a veces mediante estructuras ecoicas). Hacia el paciente, la acompañante en ocasiones corrige o complementa la información de 
este, pero principalmente se dirige a él para explicarle aspectos de la enfermedad (ocupando una función que habría correspondido a M), y para transmitirle ánimos. Por su parte, las escasas intervenciones del paciente consisten en aportar alguna información sobre sus síntomas y en preguntar por una eventual operación que no ha sido mencionada por la médica. La reacción de la doctora ante el acaparamiento de la palabra y el manejo de la situación de la acompañante, así como ante la pasividad del paciente, parece ser de aceptación de esos roles discursivos, pues excepto en alusiones directas a $\mathrm{P}$ en cuanto a síntomas o indicaciones de pruebas, no es él el destinatario único de su discurso, sino que ambos ( $\mathrm{P}$ y A) son codestinatarios directos, y en ocasiones (por ejemplo cuando receta medicamentos), su destinataria es únicamente A. De esta forma, M parece dejarse llevar por la inercia de la interacción hasta el punto de que en dos ocasiones usa la segunda persona del plural para dar la instrucción para realizar una prueba médica (beber agua) que solo atañe a P:

\section{(3) [Núñez 4E]}

1 M: primero bebéis así dos o tres vasos de agua/ y luego yo te hago la ecografía/ ¿vale?

2 A: muy bien

El uso de la forma plural del verbo por parte de la doctora puede entenderse por un fin instrumental: encargarle a A de la preparación adecuada de $\mathrm{P}$ para la prueba (que beba suficiente agua), confirmando de esta forma a A el rol de enfermera y cuidadora. Pero además, es una forma cortés hacia $\mathrm{P}$ al incluirlo en la instrucción de la prueba (en lugar de dirigirse solo a A diciendo que beba agua), respetando así su imagen de autonomía (cf. Bravo, 2002), en este caso, el deseo del paciente de mostrarse como una persona capacitada para realizar una prueba médica.

Veamos más exactamente qué roles desempeña esta hablante a lo largo de la consulta (tabla 2, en página siguiente).

Como en la consulta anteriormente analizada, aparecen los roles esperables de secretaria, enfermera / cuidadora y conocedora de temas médicos. Pero interesa destacar dos nuevos tipos de roles. Por una parte, uno asociado al de secretaria, en cuanto a gestión de asuntos prácticos relacionados con la situación, y al de portavoz, en cuanto a que asume una función de reemplazar a P en la comunicación, o reportera, pues toma la iniciativa por cuenta propia. Sin embargo, se trata de un caso especial de sustitución al paciente, pues tiene que ver con la información de datos personales (y por tanto, aspectos ligados a la identidad personal) de un paciente presente y capacitado para informar él mismo de esos datos básicos. Por ello este rol, que se podría considerar de tipo intrusivo, lo he denominado acompañante sustituta del paciente y puede verse en el siguiente extracto. 
(4) [Núñez 4E]

1 M: entonces/vamos a poner aquí / nombre/ es

2 P: [Juan Antonio García]

3 A: [Juan Antonio García Rivera]

4 M: Juan-Antonio-García

5 A: Rivera [con uve]

6 P: [o sea que por sistema/] ellos van a lo seguro

7 A: tú tranquilo/ que no va a pasar nada [damos un poco más de ((paseo)) y ya está]

8 P: [iqué va!/] si yo no le tengo miedo a la guerra

9 M: ya lo sé/ pero es así// ¿teléfono móvil?

10 A: el seis dos ocho/ cuatro seis/ [cuatro cuatro=]

$11 \mathrm{P:} \quad$ [sabes tú ahí / ¿eh?]

12 A: =nueve dos // ipues cómo le dé el tuyo / lo llevan claro! ¡no está nunca con él!

TABLA 2. Roles identificados en el comportamiento comunicativo $\mathrm{del} / \mathrm{de}$ la acompañante protagonista

\begin{tabular}{|c|c|c|}
\hline $\begin{array}{l}\text { Tipos } \\
\text { de roles }\end{array}$ & $\begin{array}{c}\text { Ejemplos } \\
\text { de comportamiento }\end{array}$ & $\begin{array}{l}\text { Número de ocasiones } \\
\text { en que se realiza } \\
\text { el comportamiento }\end{array}$ \\
\hline Secretaria & Preguntas sobre pruebas y medicinas & 5 \\
\hline $\begin{array}{l}\text { Enfermera } \\
\text { y cuidadora }\end{array}$ & $\begin{array}{l}\text { Informar sobre síntomas, } \\
\text { análisis previos y medicación }\end{array}$ & 6 \\
\hline $\begin{array}{l}\text { Conocedora } \\
\text { de temas } \\
\text { médicos }\end{array}$ & $\begin{array}{l}\text { Opinar sobre: } \\
\text { - el grado de importancia de la } \\
\text { enfermedad } \\
\text { - los pasos que hay que seguir } \\
\text { - las condiciones para realizar las } \\
\text { pruebas }\end{array}$ & 5 \\
\hline $\begin{array}{l}\text { Asistente } \\
\text { emocional }\end{array}$ & $\begin{array}{l}\text { - Dar expresiones de ánimo } \\
\text { - Generalizar el problema } \\
\text { (hablando de otros casos parecidos) } \\
\text { - Restar importancia al tratamiento } \\
\text { (por medio de estrategias } \\
\text { de atenuación) } \\
\text { - Cambio de tema hacia temas } \\
\text { cotidianos alejados de lo médico }\end{array}$ & 8 \\
\hline $\begin{array}{l}\text { Sustituto/a } \\
\text { del paciente }\end{array}$ & $\begin{array}{l}\text { - Informar de los datos personales } \\
\text { de P (nombre y edad) } \\
\text { - Dar su número de teléfono } \\
\text { en lugar del de P }\end{array}$ & 3 \\
\hline
\end{tabular}


Como vemos, A interviene dos veces (turno 3, paralelamente a $\mathrm{P}$, y 5) para dar los datos personales de $\mathrm{P}$, ante lo que él no reacciona, pero sí cuando A responde dando su propio número de teléfono, lo que parece molestar a $\mathrm{P}$ (turno 11). Se trata del único caso en la consulta en que $\mathrm{P}$ parece quejarse del protagonismo de A, lo que supone que habría percibido la intervención de A para reemplazarle a la hora de dar sus datos personales y el número de teléfono (en 10) como descortesía por amenaza a su imagen de autonomía. La acompañante rechaza el comentario (turno 12) justificando su intervención por una cuestión práctica, lo que a su vez supone un enfoque en su propia imagen de autonomía.

El otro rol destacado en la tabla (aparece, mediante diferentes comportamientos comunicativos, en ocho ocasiones durante toda la consulta) es el que denomino rol de asistente emocional, como se ve en la siguiente secuencia, inmediatamente posterior a la comunicación de los resultados médicos.

(5) [Núñez 4E]

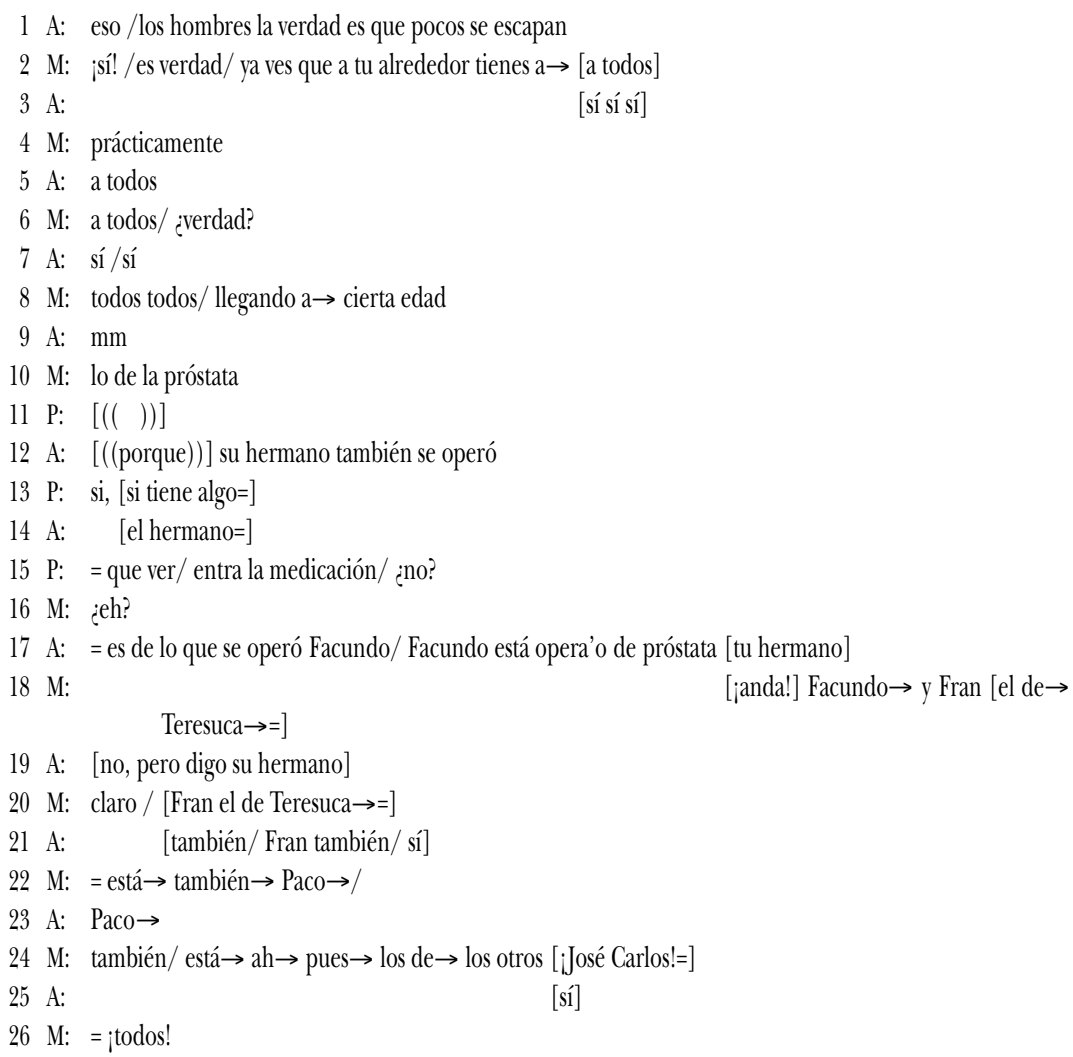


$27 \mathrm{~A}: \quad$ todos /sí/sí

28 M: es así / (es) llegando a cier- $\rightarrow$ tampoco es una cosa que dé mucha $\rightarrow$ problema/ la verdad

29 A: bueno/ lo que haya que hacer /se hace

En esta secuencia se desencadena una serie de intervenciones de la médica y la acompañante, lo que supone una verbalización de la noticia que sirva para asimilarla, si bien solo A participa en ello. Las estrategias iniciadas por A incluyen la generalización mediante la afirmación de que el cáncer de próstata es una enfermedad frecuente (en el turno 1) y la ejemplificación con personas conocidas que lo han padecido a lo largo de los turnos siguientes. Estas iniciativas de A son respaldadas por $\mathrm{M}$ en sus respuestas. P, por su parte, solo reacciona para preguntar por el tratamiento que le espera (13 y 15), un asunto que repite cinco veces más durante el resto de la consulta, lo que, teniendo en cuenta sus escasas palabras, muestra su preocupación ${ }^{9}$. Mediante las generalizaciones, se entiende que A está asumiendo un papel asociado a su rol de cuidadora y enfermera, que además de ocuparse de lo práctico y cuidarle físicamente, también tiene como función vigilar por el estado psicológico de $\mathrm{P}$, lo que concluye con una sentencia (turno 29) donde asume la función de empujar a $\mathrm{P}$ a afrontar su futuro. Se trata de un comportamiento de ayuda al otro que repercute en la imagen afiliativa (Bravo, 2002) de ambos interactuantes: por parte de $\mathrm{P}$ la de sentirse apoyado por alguien muy próximo social y emocionalmente a él; por parte de A la de cumplir con una de las funciones esperadas para su rol de cuidadora (unido aquí a su rol de cónyuge): la de apoyar emocionalmente al otro ${ }^{10}$.

Esta misma actitud se repite en la secuencia en que $\mathrm{P}$ y A se quedan a solas (la doctora sale unos momentos de la consulta) y A aprovecha para hablar de las actividades previstas cuando salgan del centro de salud.

(6) [Núñez 4E]

$1 \mathrm{~A}:$ bueno/ tenemos que parar en el tanatorio/ ¡bueno!/ coger las medicinas/ quería yo mirar $\rightarrow$ la lotería del mes/ de la semana pasada y echarla// jahora lo hacemos!/// hoy viene a comer Marta y el niño// porque tuvo que llevar la madre a la niña al médico/ que le lastimó en un brazo// pero a la tarde va a dar clase

2 P: ¿esto qué $\rightarrow$ ? ¿que es? ¿tiene complicaciones la $\rightarrow$ ? ¿es?

$3 \mathrm{~A}:$ § jes la próstata Juan! jno tiene nada! casi todos los hombres de mayores lo tenéis// casi todos§

${ }^{9}$ Comenta Cordella (2011a: 484) que en las consultas oncológicas analizadas por ella, el silencio de los pacientes puede ser "reflejo de la carga emocional que sienten con la situación que están viviendo".

${ }^{10}$ Cordella (2011a: 484) añade esta función de ofrecer empatía a otras de carácter práctico en el rol de asistente familiar de salud. 


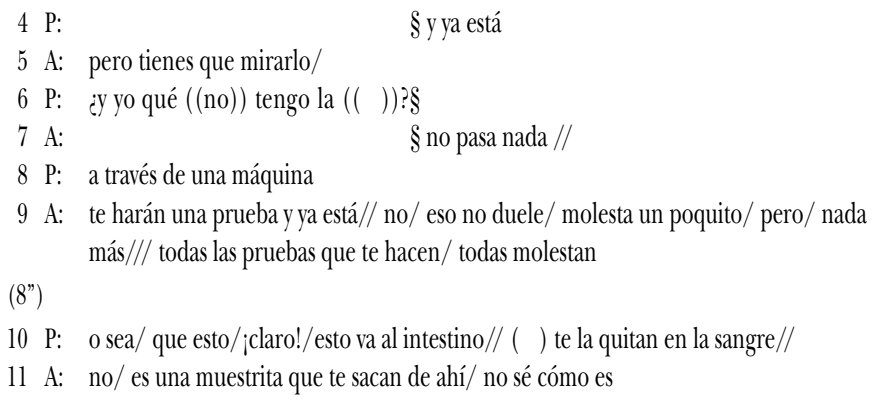

Como vemos, A continúa con su rol de asistente emocional con el uso de las mencionadas estrategias de generalización (turno 3), minimización del problema mediante recursos pragmalingüísticos de atenuación ( 7,9 y 11) e introduciendo una nueva estrategia (en el turno 1) consistente en cambiar de tema para hablar de otros cotidianos: visitar un tanatorio, comprar medicamentos, continuar con su costumbre de jugar a la lotería, anunciar la visita de Marta (posiblemente un familiar) y narrar lo que le ha ocurrido a esa persona. Es una estrategia que puede ayudar a apartar el tema de la enfermedad de la mente del paciente, desdramatiza la enfermedad y la normaliza, integrándola en la vida cotidiana. Se trata de una estrategia que protege la imagen afiliativa del destinatario (Bravo, 2002), mostrando cercanía, apoyo y consideración al otro por su malestar.

\section{DisCuSión DE RESUltAdOS Y CONCLUSIÓN}

El presente estudio ha tratado de mostrar la importancia del papel del acompañante en la consulta médica de atención primaria, un aspecto ya destacado por estudios tanto médicos, como pragmáticos. La importancia de este papel viene dada, en primer lugar, por la multiplicidad de roles que este interactuante puede desempeñar. Los más evidentes, y descritos en la literatura sobre el tema, los relacionados con el cuidado (rol de enfermero y cuidador) y con lo práctico (rol de secretario), en un momento en que el paciente requiere de este tipo de apoyo para su tratamiento. Además, el rol de conocedor de temas médicos supone una función que cuando se desarrolla en colaboración con el médico (como en los casos vistos), sirve como puente entre el facultativo y el paciente, facilitando la asimilación de la información sanitaria por parte de este.

Pero, además, el análisis ha mostrado otros roles no vinculados a cuestiones prácticas e instrumentales, como los anteriores, sino a cuestiones sociales y psicológicas.

Con una función social, el rol de dinamizador social, manifestado en la interacción analizada en la narración de anécdotas, pequeñas discusiones 
de temas fuera del ámbito sanitario y el uso del humor, puede a priori parecer superfluo en este tipo de situación; sin embargo, contribuye a establecer la cercanía entre médico, paciente y su acompañante, lo que fortalece la afiliación, y así la confianza que los pacientes (y por extensión sus acompañantes familiares) desean tener con su médico. Por otra parte, el desempeño de este rol puede aportar información al médico sobre la situación psicosocial del paciente, un factor relevante en la atención primaria, como se comentó más arriba ${ }^{11}$.

Con una función psicológica, el rol de asistente emocional va más allá de las actuaciones esperadas en el rol de enfermero y cuidador, pues se refiere a apoyar anímicamente al paciente, y para ello el acompañante debe tener una relación de cercanía social y/o afectiva con este. Comportamientos comunicativos como el cambio de tema, la introducción en la conversación de asuntos cotidianos no ligados al de la enfermedad, el uso de la generalización y la atenuación en los comentarios en torno al tratamiento y las expresiones de ánimo, son estrategias discursivas que contribuyen a reforzar lo anímico en el proceso de curación del paciente.

Otro rol que llama la atención, por la posible amenaza a la imagen social del paciente, es el de sustituto del paciente, donde el acompañante puede llegar a suplantar innecesariamente a aquel en situaciones en que el paciente se puede arreglar. Siguiendo el planteamiento de estudios sociopragmáticos del español en torno a la imagen social (ver, por ejemplo, Hernández Flores, 2016), la amenaza iría hacia su imagen de autonomía (su capacidad para realizar acciones por sí mismo). Sin embargo, opino que esta valoración debe ser cuestionada o matizada en situaciones de comunicación médica en torno a una enfermedad grave y/o a pacientes en condiciones físicas o anímicas limitadas, como es el caso de esta consulta, con un paciente que padece párkinson y con sospecha de cáncer, y que anímicamente, por la escasez de sus intervenciones, y sus preguntas sobre el tratamiento, parece preocupado. En este caso, las posibles amenazas a la imagen de autonomía por parte de la acompañante al intervenir en todo lo referente a su persona tal vez habría que considerar que pueden quedar bloqueadas. Por otra parte, las características mostradas por la acompanante, una persona muy práctica con control tanto sobre los asuntos médicos como de la vida diaria, y acostumbrada a afrontar las enfermedades

${ }^{11}$ La confianza en su médico es el valor más mencionado por los informantes de una encuesta realizada en el mismo centro de atención primaria donde se grabó el corpus de este trabajo. Por su parte, los médicos de dicho centro destacaron en entrevistas la importancia que otorgaban a tener tiempo para dialogar con el paciente y llegar a conocerlo, y así alcanzar un diagnóstico y una prescripción más precisos. 
de sus allegados, se complementan con lo que quizás el paciente requiera en esos momentos ${ }^{12}$.

Por ello, en este caso, ambos papeles parecen complementarse de acuerdo con la situación, y esta distribución de roles es aceptada y confirmada por la médica según lo que se desprende de sus intervenciones comunicativas.

Por otra parte, la complementación de roles también se observa en la primera consulta analizada, donde la narración de temas privados muestra lo que paciente y acompañante comparten en su vida diaria: el cuidado de los nietos, los viajes o los partidos de fútbol por televisión, de forma que la enfermedad de ella es también un asunto compartido. De esta manera, en la consulta el acompañante complementa la información de la paciente cuando esta tiene dificultades de hacerlo debido a su tos, o es el encargado de recordar al médico la exploración de bronquios que ella necesita. En ese sentido, su función no es sustituir a la paciente, sino complementar sus intervenciones, lo que lo confirma como acompañante coparticipativo. Estas observaciones muestran que los roles de paciente y acompañante son interdependientes y que los rasgos de uno se configuran en función de los del otro.

En relación con la imagen social de los interactuantes, el rol de dinamizador social permite construir una relación tripartita (paciente-médicoacompañante) que facilita la comunicación y reafirma la imagen afiliativa de los interactuantes. En el rol de asistente emocional se refuerza la afiliación mediante el sostén emocional.

Por último, desde la perspectiva del análisis discursivo, es interesante senalar que secuencias como la de paciente y acompañante sin la presencia de la médica muestran cómo estos dos primeros roles continúan su construcción fuera del marco de la interacción con el médico, y confirman, como ha señalado la investigación reciente, que el estudio de la comunicación medico-paciente se puede ver enriquecida con la incorporación al análisis de otros marcos comunicativos.

12 Cordella (2011a) recoge que en las consultas oncológicas hay pacientes a quienes las intervenciones activas del acompañante los tranquiliza. 


\section{BIBLIOGRAFÍA}

BACKHAUs, P. (2009): "Politeness in institutional elderly care in Japan: A crosscultural comparison", Journal of Politeness Research. Language, Behaviour, Culture, 5(1), págs. 53-72.

Bravo, D. (2002): "Actos asertivos y cortesía: Imagen del rol en el discurso de académicos argentinos”, en D. Bravo y M. E. Placencia (eds.), Actos de habla y cortesía en el español, Munich, Lincom Europa, págs.141-174.

BRAVO, D. (2005): "Categorías, tipologías y aplicaciones: hacia una redefinición de la cortesía comunicativa”, en D. Bravo (ed.), Estudios de la (des)cortesía en español. Categorías conceptuales y aplicaciones a corpora orales y escritos, Buenos Aires, Dunken, págs. 21-52.

Brown, B. y CrawFord, P. (2009): "Politeness strategies in question formulation in a UK telephone advisory service", Journal of Politeness Research. Language, Behaviour, Culture, 5(1), págs. 73-92.

CePEdA, G. (2005): "Cortesía, imagen social y aceptación del mensaje terapéutico. Modalización en el discurso de entrevista clínica”, en D. Bravo (ed.), Estudios de la (des)cortesía en español, Estocolmo/Buenos Aires, Programa EDICE/ Dunken, págs. 163-187.

Cepeda, G. (2006): "La voz empática médica y las estrategias de cortesía verbal”, Estudios Filológicos, 41, págs. 55-69.

CEPEDA, G. (2009): "La justificación como efecto de cortesía en la entrevista clínica”, en D. Bravo, N. Hernández Flores y A. Cordisco (eds.), Aportes pragmáticos, sociopragmáticos y socioculturales a los estudios de la cortesía en español, Estocolmo/Buenos Aires, Programa EDICE/Dunken, págs. 199-230.

Cordella, M. (2011a): "Enfrentándose al cáncer en compañía: el rol del familiar en la consulta oncológica”, Discurso \& Sociedad, 5(3), págs. 469-491.

Cordella, M. (2011b): "A triangle that may work well: Looking through the angles of a three-way exchange in cancer medical encounters", Discourse \& Communication, 5(4), págs. 337-353.

Goffman, E. (1967): Interactional ritual: Essays face-to-face behaviour, New York, Pantheon Books.

HARrison, S. y BARLow, J. (2009): "Politeness strategies and advice-giving in an online arthritis workshop", Journal of Politeness Research. Language, Behaviour, Culture, 5(1), págs. 93-112.

HeRnández Flores, N. (2013): "Actividad de imagen: caracterización y tipología en la interacción comunicativa”, Pragmática Sociocultural, 1(2), págs. 175-198.

Hernández Flores, N. (2016): "Modalidad y direccionalidad del efecto social en la interacción hablada. Una discusión e ilustración de actividades de imagen en la interacción médico-paciente”, en D. Dumitrescu y D. Bravo (eds.), Roles situacionales, interculturalidad y multiculturalidad en encuentros en español, Buenos Aires/Los Ángeles, Dunken/Academia Norteamericana de la Lengua, págs. 193-211. 
Hernández Flores, N. y Rodríguez Tembrás, V. (en prensa): “'Lo que se debe hacer es cambiar un poco el estilo de vida': estrategias de atenuación del consejo en un consultorio médico", en M. Albelda (ed.), Estrategias atenuantes en géneros discursivos del español: interfaz semántico-pragmática. Spanish in context.

HERNÁNDEz LóPEz, M. (2009): La gestión de las relaciones interpersonales en la interacción médico-paciente: estudio contrastivo inglés británico-español peninsular. Tesis doctoral. Sevilla, Universidad de Sevilla.

HERnÁNDEZ LóPEz, M. (2010a): "Principios sociopragmáticos de la interacción y dinamismo de las relaciones de poder entre médico y paciente", en F. Orletti y L. Mariottini (eds.), Descortesía en español. Espacios teóricos y metodológicos para su estudio, Roma/Estocolmo, Università RomaTre/Programa EDICE, págs. 655-675.

HeRnÁNDEz LóPEz, M. (2010b): "El humor como lengua de contacto ¿estrategia afiliativa o de auto-afirmación?: estudio comparativo inglés-español del discurso institucional”, Odisea, 11, págs. 258-299.

López García-Ramos, L., Martín, D. L., Jiménez, F. J. B., De Colosía Civantos, M. P. y González, M. P. (2009): "Influencia del acompañante en la negociación y la duración de la consulta en atención primaria”, Atención Primaria, 41 (3), págs. 147-151.

MADFES, I. (2003): "La confrontación de imágenes en una interacción asimétrica: ¿Médico y paciente: Afiliación o conflicto?”, en D. Bravo (ed.), Actas del Primer Coloquio Internacional del Programa EDICE. La perspectiva no etnocentrista de la cortesía: identidad sociocultural de las comunidades hispanohablantes, págs. 172-185.

Madfes, I. (2006): "Polifonía en la entrevista médica. El acompañante o el pariente pobre de la interacción médico-paciente", Oralia, 9, págs. 167-184.

Mullany, L. (2009): "Introduction: Applying politeness research to health care communication”, Journal of Politeness Research. Language, Behaviour, Culture, $5(1)$, págs. 1-10.

Oliveira, S. M. DE y Hernández Flores, N. (2014): Corpus de interacción médicopaciente en Galicia (no publicado).

Pennbrant, S. (2013): "A trustful relationship - the importance for relatives to actively participate in the meeting with the physician", International Journal of Qualitative Studies on Health and Well-being, 8: 20608.

Rodríguez Tembrás, V. (2016): "Alternancia de lenguas como estrategia de actividad de imagen en la comunicación médico-paciente en un consultorio gallego", Textos en Proceso, 2(1), págs. 94-121.

Turabián, J. L. y Pérez Franco, B. (2015): "El acompañante en la entrevista clínica de atención primaria”, SEMERGEN-Medicina de Familia, 41(4), págs. 206-213.

Turabián, J. L., Minier-Rodríguez, L. E., Moreno-Ruiz, S., Rodríguez-Almonte, F. E., y Cucho-Jove, R. (2016): "Types of Companion of the Patient in Family Medicine”, J Health Edu Res Dev, 4: 186. 